\title{
High expression of ubiquitin-conjugating enzyme E2A predicts poor prognosis in hepatocellular carcinoma
}

\author{
JIAN-DONG SHEN ${ }^{1 *}$, SHOU-ZHONG FU ${ }^{1 *}$, LIN-LING JU ${ }^{2}$, YI-FANG WANG ${ }^{2}$, FENG DAI ${ }^{1}$, \\ ZHAO-XIU LIU ${ }^{3}$, HAN-ZHENG JI ${ }^{4}$, JIAN-GUO SHAO ${ }^{5}$ and ZHAO-LIAN BIAN ${ }^{5}$
}

\begin{abstract}
${ }^{1}$ Department of Invasive Technology; ${ }^{2}$ Nantong Institute of Liver Diseases, Nantong Third People's Hospital, Nantong University, Nantong, Jiangsu 226006; ${ }^{3}$ Department of Gastroenterology, Affiliated Hospital of Nantong University, Nantong, Jiangsu 226021; ${ }^{4}$ Library of Nantong Third People's Hospital; ${ }^{5}$ Department of Gastroenterology and Hepatology, Nantong Institute of Liver Diseases, Nantong Third People's Hospital, Nantong University, Nantong, Jiangsu 226006, P.R. China
\end{abstract}

Received August 16, 2017; Accepted February 1, 2018

DOI: $10.3892 / 01.2018 .8189$

\begin{abstract}
The present study aimed to illustrate the association of the expression of ubiquitin-conjugating enzyme E2A (UBE2A) with the clinicopathological parameters and prognosis in hepatocellular carcinoma (HCC). The expression levels of UBE2A mRNA and protein in a total of 276 HCC tissues and six liver cell lines was detected by fluorescent quantitative polymerase chain reaction, western blotting and immunohistochemistry. Statistical analysis was also performed to assess the association of the expression of UBE2A with the clinicopathological parameters and prognosis by the GraphPad Prism and SPSS version 21.0 software. UBE2A mRNA and protein were highly expressed in HCC tissues compared with those in the adjacent normal tissue. Immunohistochemical analysis revealed that UBE2A protein was more strongly stained in the 276 paraffin-embedded HCC tissues as compared with the 63 adjacent normal tissue. Statistical analysis also demonstrated that UBE2A expression was significantly associated with histological differentiation, TNM stage and vascular invasion of $\mathrm{HCC}(\mathrm{P}<0.05)$. Notably, HCC patients with a high expression of UBE2A had a shorter survival time as compared with those with a low expression of UBE2A. There results suggested that UBE2A may be involved in the pathogenesis of $\mathrm{HCC}$ and may serve as an important prognostic marker.
\end{abstract}

Correspondence to: $\mathrm{Dr}$ Zhao-Lian Bian, Department of Gastroenterology and Hepatology, Nantong Institute of Liver Diseases, Nantong Third People's Hospital, Nantong University, 60 Middle Qingnian Road, Nantong, Jiangsu 226006, P.R. China

E-mail: bianzhaolian1998@ntu.edu.cn

"Contributed equally

Abbreviations: UBE2A, ubiquitin-conjugating enzyme E2A; HCC, hepatocellular carcinoma; AFP, $\alpha$-fetoprotein

Key words: ubiquitin-conjugating enzyme E2A, prognosis, hepatocellular carcinoma, biomarker
Further exploration of the involvement of UBE2A in HCC development may provide novel therapeutic targets.

\section{Introduction}

Hepatocellular carcinoma (HCC) is the third leading cause of cancer-associated mortality worldwide and the fifth most common cancer type (1). HCC is a global health burden and its prevalence varies worldwide (2). The incidence rate is reported to be higher in Asia (>20 cases/100,000 individuals) in comparison with that in North America and Europe ( $<5$ cases/100,000 individuals) (3). The prognosis of HCC is poor, as evident by the fact that the number of annual mortality cases $(\sim 600,000)$ is almost equal to the number of new cases diagnosed annually (4). The median rate for 1-year survival is $80 \%$ (range, 63-97\%), for 3-year survival is $70 \%$ (range, $34-78 \%$ ) and for 5-year survival is 50\% (range, 17-69\%) (5). Surgery remains the most important treatment strategy for patients with HCC. The 5-year survival rate of patients with early HCC subsequent to resection treatment reaches $50 \%$, although these patients exhibit a high recurrence rate $(6,7)$. Despite advances in the diagnosis (8) and treatment (9) of HCC, the prognosis of this disease remains poor (10). Therefore, further clarification of the mechanisms underlying its development is important $(11,12)$.

At present, the prognosis of HCC is predicted based on imaging findings and biomarkers. Various biomarkers, including $\alpha$-fetoprotein (AFP) (13), des- $\gamma$-carboxy prothrombin (14) and $\alpha$-1-fucosidase (15), have been identified over the past three decades. However, accurate indicators for the prognosis of HCC are limited. Consequently, there is an urgency to develop a novel biomarker for the prognosis of HCC.

The ubiquitin system serves a critical role in numerous cellular events, including the cell cycle regulation, DNA repair, stress responses, metabolic homeostasis, organelle biosynthesis and apoptosis $(16,17)$. A recent study demonstrated that the hepatitis $\mathrm{C}$ virus NS3 protein enhanced HCC cell invasion by promoting protein phosphatase magnesium-dependent 1A ubiquitination and degradation (18), which indicated degradation and ubiquitination of proteins were involed in 
the deveopment of HCC. Ubiquitination is catalyzed by a series of enzymes, including ubiquitin-activating enzyme E1, ubiquitin-conjugating enzyme E2A (UBE2A) and E3 ubiquitin ligase (19). UBE2A is a member of a group of E2 enzymes (20) that are involved in DNA damage repair by catalyzing the ubiquitination of different target proteins (21). DNA methylation involves the attachment of a methyl group to the cytosine, guanine or amino acids of histones wrapped with DNA, often leading to normal or aberrant modification in gene function (22). Previous studies have revealed that UBE2A is considered as a node for directing different pathways of DNA damage repair (23), and UBE2A is overexpressed in certain cancer cell lines and tumors (24). However, the role and function of UBE2A in HCC remain unknown. Therefore, the present study aimed to investigate the significance of UBE2A expression and its prognostic value in HCC.

\section{Materials and methods}

Patients and tumor tissues. A total of 276 HCC tissue samples and 63 adjacent normal tissue were obtained from 276 patients with HCC undergoing resection treatment in the Nantong Third People's Hospital (Nantong, China) and the Affiliated Hospital of Nantong University (Nantong, China) between September 2007 and December 2014. The 276 participants included 152 male and 124 female patients, aged between 26 and 80 years (mean age, 53.4 years). The distance between the para-carcinoma and carcinoma tissue was $>2.5 \mathrm{~cm}$. All the tissues were fixed in $10 \%$ neutral buffered formalin for $24 \mathrm{~h}$ and then embedded in paraffin. A total of 42 paired tumor and adjacent normal tissues in all these patients were immediately frozen at $-80^{\circ} \mathrm{C}$ until use. The diagnosis of all HCC patients was confirmed according to HCC guidelines for the diagnosis and treatment of European Association (25), and no patient received chemotherapy or radiotherapy prior to surgery. This study was approved by the Ethics Committee of Nantong Third People's Hospital, and all the patients provided signed informed consent.

Cell lines and culture. Human HCC cell lines, including HepG2 (cat. no. TCHu72), Huh-7 (cat. no. TCHu182), Bel-7402 (cat. no. TCHu10), and Bel-7701 (cat. no. TCHu42) cells, and the human normal liver cell line HL-7702 (cat. no. GNHu6) were obtained from the Cell Bank of the Chinese Academy of Sciences (Shanghai, China). The SNu-423 was a gift from Mr. Xiangwei Hua from Renji Hospital, School of Medcine, Shanghai Jiaotong University (Shanghai, China) and stored in the laboratory of the Nantong Institute of Liver Diseases (Nantong, China). The cells were cultured in Dulbecco's modified Eagle's medium (Invitrogen; Thermo Fisher Scientific, Inc., Waltham, MA, USA) supplemented with $10 \%$ fetal bovine serum (Cell Sciences, Inc., Newburyport, MA, USA), $100 \mathrm{mg} / \mathrm{ml}$ streptomycin and $100 \mathrm{U} / \mathrm{ml}$ penicillin (Invitrogen; Thermo Fisher Scientific, Inc.) in a humidified incubator with $5 \% \mathrm{CO}_{2}$ at $37^{\circ} \mathrm{C}$.

RNA isolation and fluorescent quantitative polymerase chain reaction $(q P C R)$. Total RNA was isolated from the tissues and cells using RNAiso Plus (Takara Biotechnology Co., Ltd., Dalian, China). The quality and quantity of the isolated RNA were evaluated using a UV-1800 spectrophotometer (Shimadzu Corporation, Kyoto, Japan). Next, total RNA was converted into cDNA using a PrimeScript RT Reagent kit (Perfect Real Time; Takara Biotechnology Co., Ltd.). qPCR was performed using a SYBR-Green PCR Master Mix (Vazyme Biotech Co., Ltd., Nanjing, China). The sequences of primers used in the qPCR analysis were as follows: $\beta$-actin forward, 5'-GGACTT CGAGCAAGAGATGG-3' and reverse, 5'-AGGAAGGAA GGCTGGAAGA-3'; UBE2A forward, 5'-CCTATGATGTGT CTTCCATTCTAAC-3' and reverse, 5'-TATTCCCGTTTG TTCTCCTG-3'. qPCR was initially performed at $95^{\circ} \mathrm{C}$ for $5 \mathrm{~min}$, and then samples were subjected to 40 cycles of amplification at $95^{\circ} \mathrm{C}$ for $10 \mathrm{sec}$ and at $60^{\circ} \mathrm{C}$ for $30 \mathrm{sec}$. The mRNA expression of UBE2A was normalized to that of $\beta$-actin. The fold amplification for each gene was calculated using the $2^{-\Delta \Delta C q}$ method (26).

Western blotting. Approximately $25 \mathrm{mg}$ HCC tissue was lysed with RIPA lysis buffer including phosphatase inhibitor cocktail (Beyotime Institute of Biotechnology, Shanghai, China). The supernatant was then harvested after $10,000 \mathrm{x}$ g centrifugation for $10 \mathrm{~min}$ in $4^{\circ} \mathrm{C}$, and then protein concentration was measured using a BCA Protein Assay kit (Beyotime Institute of Biotechnology). Equal amounts of protein were electrophoretically separated by $10 \%$ SDS-PAGE and transferred onto nitrocellulose membranes at $100 \mathrm{~V}$ for $90 \mathrm{~min}$. The expression of UBE2A was analyzed using a single specific primary antibody, RAD6 (cat. no. ab31917; 1:1,000; Abcam, Cambridge, MA, USA), and the $\beta$-actin (cat. no. ab8227; 1:1,500; Abcam) was used as control. Subsequent to overnight incubation at $4^{\circ} \mathrm{C}$, the membranes were incubated with horseradish peroxidase-conjugated goat anti-rabbit IgG secondary antibody (cat. no. KC-RB-035; 1:3,000; Kangchen Biotech Co., Ltd., Shanghai, China) at room temperature for $1 \mathrm{~h}$. Next, the samples were washed three times with Tris-buffered saline and Tween-20, and an enhanced chemiluminescence system (Thermo Fisher Scientific, Inc.) was used to visualize the results. Grey values of western blotting were analyzed using Image $\mathrm{J}$ software 1.48 (National Institutes of Health, Bethesda, MD, USA).

\section{Hematoxylyin and eosin (H\&E) and immunohistochem-} istry. The liver tissues were fixed in $10 \%$ neutral buffered formalin for $24 \mathrm{~h}$ at room temperature and then embedded in paraffin. The $4-\mu \mathrm{m}$ slides were stained with $0.5 \%$ hematoxylin [weight/volume (W/V)] for $10 \mathrm{~min}$ and wased with water for $10 \mathrm{~min}$, then the slides was satined with $1 \%$ eosin $(\mathrm{W} / \mathrm{V})$ for $30 \mathrm{sec}$. All the procedures were performed at room temperature. The H\&E staining kit (cat. no. C0105; Beyotime Institute of Biotechnology) was obtained from Beyotime Institute of Biotechnology. An immunohistochemical assay was performed on formalin-fixed, paraffin-embedded HCC tissues using antibodies against UBE2A (27). Briefly, $4 \mu \mathrm{m}$ slides were deparaffinized through a series of xylene baths and graded alcohols. Antigen retrieval was conducted by microwaving the slides in citrate buffer ( $\mathrm{pH} \mathrm{6.0)} \mathrm{for} 10 \mathrm{~min}$. The endogenous peroxidase activity was blocked by $3 \% \mathrm{H}_{2} \mathrm{O}_{2}$ for $15 \mathrm{~min}$ at room temperature, and then the sections were incubated with rabbit anti-human UBE2A (cat. no. ab31917, 1:200 dilution; Abcam) overnight at $4^{\circ} \mathrm{C}$. Subsequently, a horseradish peroxidase-conjugated anti-mouse/rabbit secondary antibody (cat. no. D-3004; 
Table I. Association between UBE2A expression and clinicopathological characteristics of patients.

\begin{tabular}{|c|c|c|c|c|}
\hline \multirow[b]{2}{*}{ Characteristic } & \multirow[b]{2}{*}{ Total } & \multicolumn{2}{|c|}{ UBE2A expression } & \multirow[b]{2}{*}{ P-value } \\
\hline & & High expression $(n=166)$ & Low expression $(n=110)$ & \\
\hline Age (years) & & & & 0.9034 \\
\hline$<53$ & 129 & 78 & 51 & \\
\hline$\geq 53$ & 147 & 88 & 59 & \\
\hline Sex & & & & 0.8049 \\
\hline Male & 152 & 90 & 62 & \\
\hline Female & 124 & 76 & 48 & \\
\hline TNM stage $^{a}$ & & & & 0.0010 \\
\hline I and II & 103 & 49 & 54 & \\
\hline III and IV & 173 & 117 & 56 & \\
\hline Histological differentiation ${ }^{\mathrm{b}}$ & & & & 0.0099 \\
\hline I and II & 144 & 76 & 68 & \\
\hline III and IV & 132 & 90 & 42 & \\
\hline Vascular invasion & & & & 0.0253 \\
\hline Negative & 157 & 85 & 72 & \\
\hline Positive & 119 & 81 & 38 & \\
\hline Tumor diameter $(\mathrm{cm})$ & & & & 0.7031 \\
\hline$<5$ & 173 & 106 & 67 & \\
\hline$\geq 5$ & 103 & 60 & 43 & \\
\hline
\end{tabular}

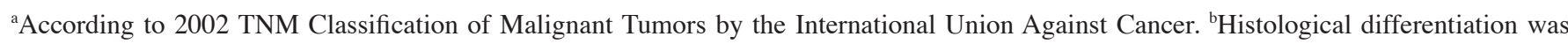
classified by the Edmondson-Steiner grade. UBE2A, ubiquitin-conjugating enzyme E2A.

Shanghai Changdao Biotechnology Co., Ltd, Shanghai, China) was applied for $30 \mathrm{~min}$ the following day at room temperature. Following DAB staining for $1 \mathrm{~min}$ at room temperature, hematoxylin staining was performed. Stained sections were observed by 1 pathologist. Five fields of each slide were randomly observed under an optical microscope in x200 magnification, and then the expression level of UBE2A was scored on a scale of 0-3 according to the intensity, as follows: 0, negative expression; 1 , weak expression; 2 , moderate expression; and 3 , strong expression.

Statistical analysis. Data are expressed as the mean \pm standard deviation. The differences between groups were determined by nalysis of variance, and comparison between two groups was conducted using the Student's t-test. The survival time was compared between HCC patients with high or low expression of UBE2A using the log-rank single factor test, and Kaplan-Meier survival curves were constructed using GraphPad Prism version 6.0 (GraphPad Software, Inc., San Diego, CA, USA). The Cox proportional hazards regression analysis was performed using the SPSS version 21.0 statistical software package (IBM Corp., Armonk, NY, USA). $\mathrm{P}<0.05$ was considered to indicate a difference that was statistically significant.

\section{Results}

Baseline characteristics. The complete clinical and follow-up data of 276 HCC patients who received no antitumor treatment prior to surgery were collected, and the cohort included 152 male and 124 female patients, aged between 26 and 80 years (mean age, 53.4 years). In total, 119 patients presented with vascular invasion, while 157 presented no vascular invasion. In addition, 144 cases had highly differentiated HCC and 132 patients had poorly differentiated HCC. The tumor diameter was $<5 \mathrm{~cm}$ in 173 patients. According to the seventh edition of the TNM Classification of Malignant Tumors (TNM-7) (28), 103 patients were classified as TNM stage I or II, while 173 were classified as stage III or IV cases. All clinical data are summarized in Table I.

Increased UBE2A expression in HCC patients and cell lines. qPCR was used to detect the expression of UBE2A in HCC and adjacent normal tissue. UBE2A mRNA expression was significantly increased in HCC tissues $(9.55 \pm 8.84)$ compared with the adjacent normal tissue $(5.74 \pm 2.25$; $\mathrm{P}<0.01$; Fig. $1 \mathrm{~A})$. Western blotting was also used to detect the level of UBE2A protein, which was observed to be highly expressed in HCC tissues, whereas it was weakly detected in adjacent normal tissue (Fig. 1B). Furthermore, immunohistochemical staining was used to detect the expression of UBE2A in HCC and adjacent normal tissue. UBE2A was expressed in the nuclei and cytoplasm of HCC cells, but it was mainly located in the cytoplasm (Fig. 1C). There was strongly positive UBE2A protein expression in the 276 HCC tissues, and weak or no expression in the 63 adjacent normal tissue, with a statistically significant difference observed ( $\mathrm{P}<0.0001$; Fig. $1 \mathrm{C}$ and $\mathrm{D})$. 

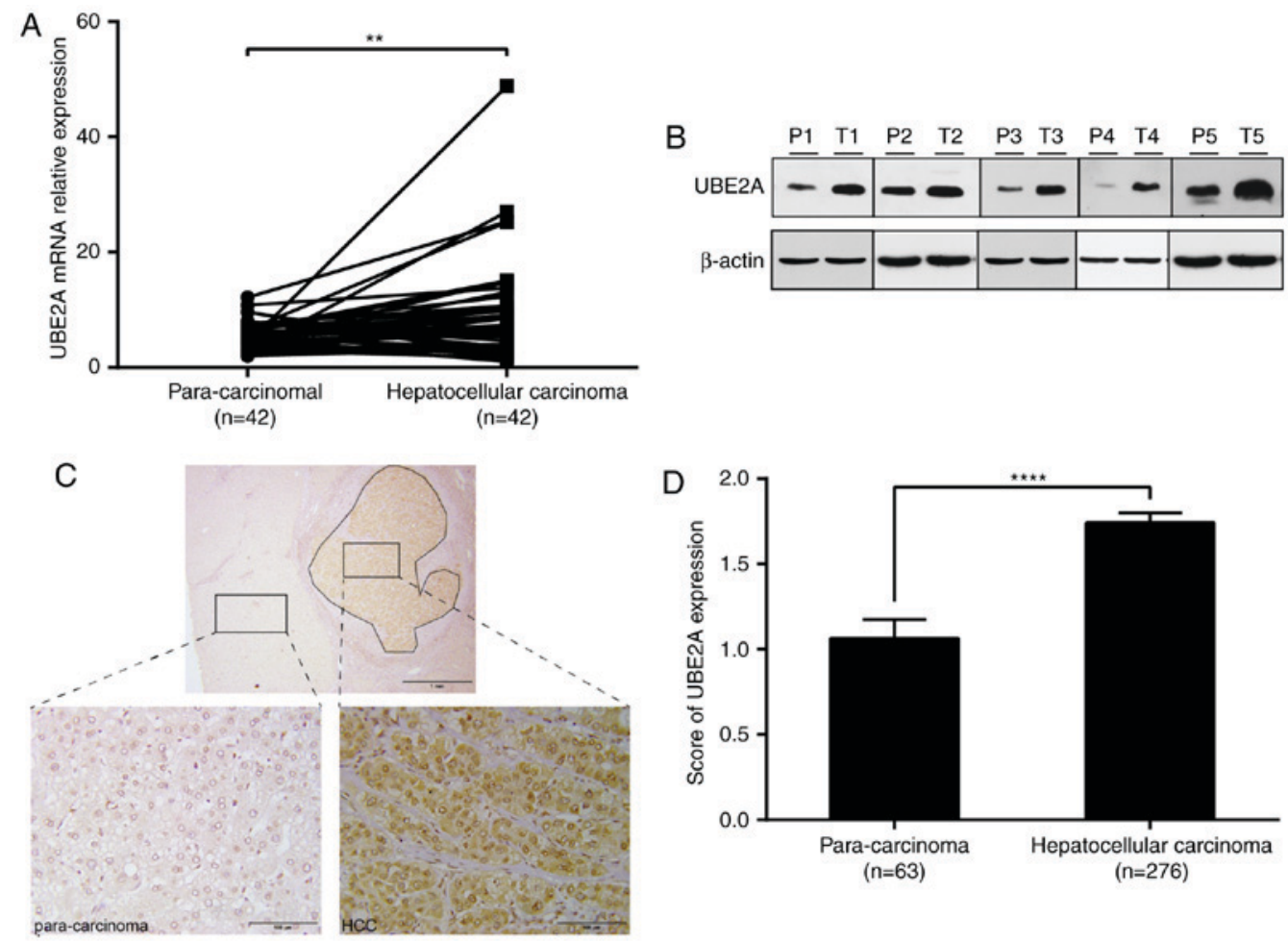

Figure 1. Expression of UBE2A in HCC and adjacent normal tissue. (A) Fluorescent quantitative polymerase chain reaction was performed for the detection of UBE2A mRNA expression in fresh HCC $(n=42)$ and adjacent normal tissue $(n=42)$. (B) Detection of UBE2A protein expression in HCC and adjacent normal tissue by western blotting. (C) Representative immunohistochemical staining of UBE2A in HCC and adjacent normal tissue. The upper image demonstrates a sample of the HCC and adjacent tissue (scale bar, $100 \mu \mathrm{m}$ ). The lower images are enlarged views of the upper image (scale bar, $100 \mu \mathrm{m})$. All images are provided at magnification, $\mathrm{x} 400$. (D) Immunohistochemical score of UBE2A expression in HCC and adjacent normal tissue. ${ }^{* * *} \mathrm{P}<0.01$ and ${ }^{* * * * *} \mathrm{P}<0.0001$. UBE2A, ubiquitin-conjugating enzyme E2A; HCC, hepatocellular carcinoma; T, tumor tissue; A, adjacent normal tissue.

UBE2A expression was then examined in various $\mathrm{HCC}$ cell lines and normal liver cells. qPCR further revealed that UBE2A mRNA was highly expressed in HCC cell lines (HePG2, 1.67 \pm 0.02 ; Huh-7, 2.56 \pm 0.15 ; Bel-7402, 2.34 \pm 0.07 ; SNu-423, 2.30 \pm 0.06 ; Bel-7701, 2.14 \pm 0.07 ) as compared with that in normal cells (HL-7702, 0.89 $\pm 0.12 ; \mathrm{P}<0.001$; Fig. 2).

Association of UBE2A expression with clinicopathological parameters of HCC patients. The correlation of UBE2A expression with the clinicopathological parameters of HCC patients was examined. A significant association was detected between UBE2A protein expression and the histological differentiation, TNM stage and vascular invasion (all $\mathrm{P}<0.05$ ). However, UBE2A expression was not associated with the age, sex and tumor size of HCC patients (Table I). According to the TNM staging system, 276 cases of HCC tissues were divided into two groups, including patients with stage I+II and stage III+IV disease. Statistical analysis of UBE2A expression in HCC patients with different TNM stage revealed that UBE2A expression in the TNM stage III+IV group was significantly higher in comparison with that in the stage I+II group $(\mathrm{P}<0.0001$; Fig. 3$)$.

Correlation of UBE2A expression with patient survival. All patients were classified into two groups according to the immunohistochemical scores as follows: A score of 0 or 1 was considered as low expression, while a score of 2 or 3 was considered as high expression. Patients in the present study were followed up for 3 to 10 years. The correlation between the

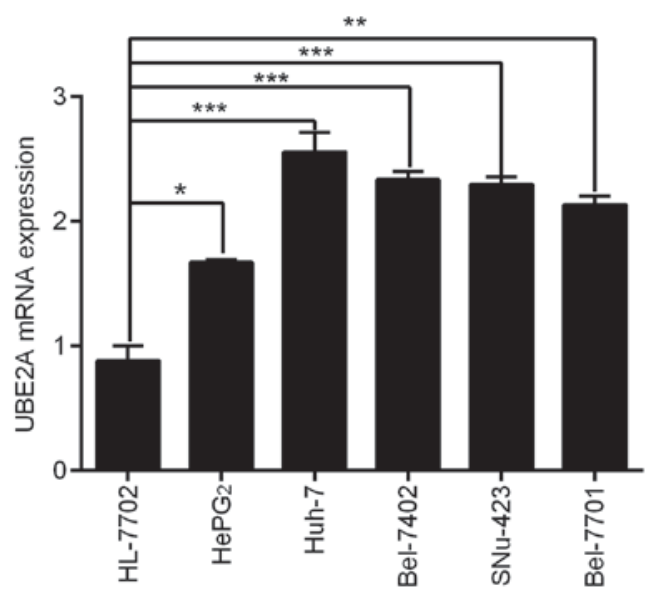

Figure 2. Detection of UBE2A mRNA expression by fluorescent quantitative polymerase chain reaction in HCC cell lines, including HepG2, Huh-7, Bel-7402, SNu-423 and Bel-7701 cells, and in normal human liver HL-7702 cells serving as the used as the control. ${ }^{*} \mathrm{P}<0.05,{ }^{* *} \mathrm{P}<0.01$ and ${ }^{* * * *} \mathrm{P}<0.001$. UBE2A, ubiquitin-conjugating enzyme E2A; HCC, hepatocellular carcinoma.

prognostic effect of UBE2A expression and the overall survival of HCC patients was then investigated. The Kaplan-Meier curve demonstrated that a high UBE2A protein level was a significant prognostic factor in revealing poor overall survival in HCC patients. The 166 patients with high UBE2A protein expression had a significantly lower survival rate as compared with the 110 patients with low UBE2A protein expression $(\mathrm{P}<0.0001 ;$ Fig. 4). In the Kaplan-Meier survival curve, a 

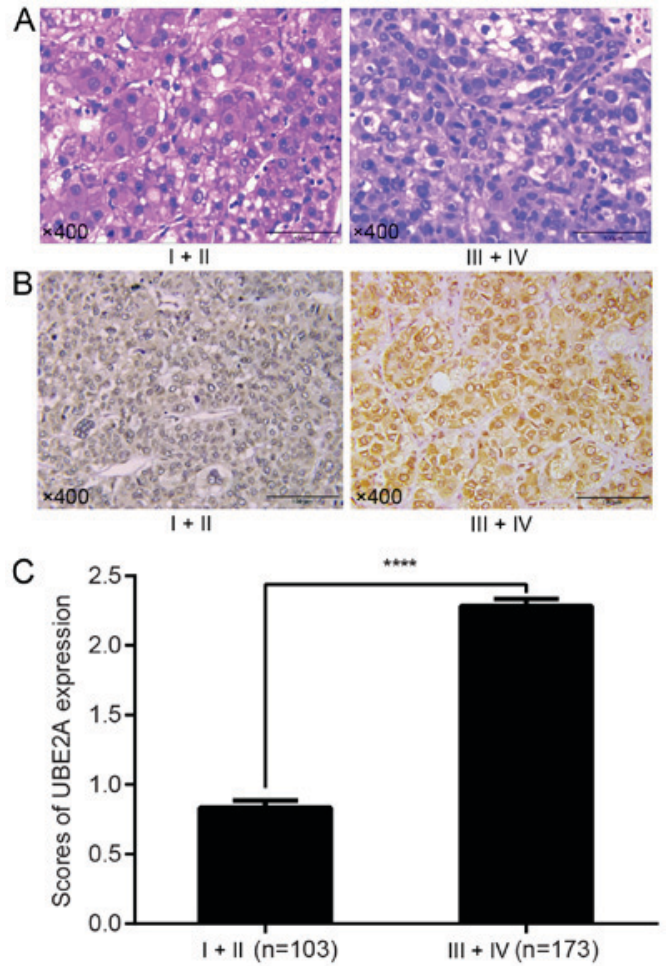

Figure 3. Correlation between UBE2A and TNM stage in HCC patients. (A) Representative hematoxylin and eosin staining of TNM stage I and IV HCC tissues (scale bar, $100 \mu \mathrm{m}$ ) and (B) representative immunohistochemical staining of UBE2A in patients with TNM stage I and IV HCC (scale bar, $100 \mu \mathrm{m})$. (C) Immunohistochemical scores of UBE2A expression in HCC patients with different TNM stages. ${ }^{* * * *} \mathrm{P}<0.0001$. UBE2A, ubiquitin-conjugating enzyme E2A; HCC, hepatocellular carcinoma.

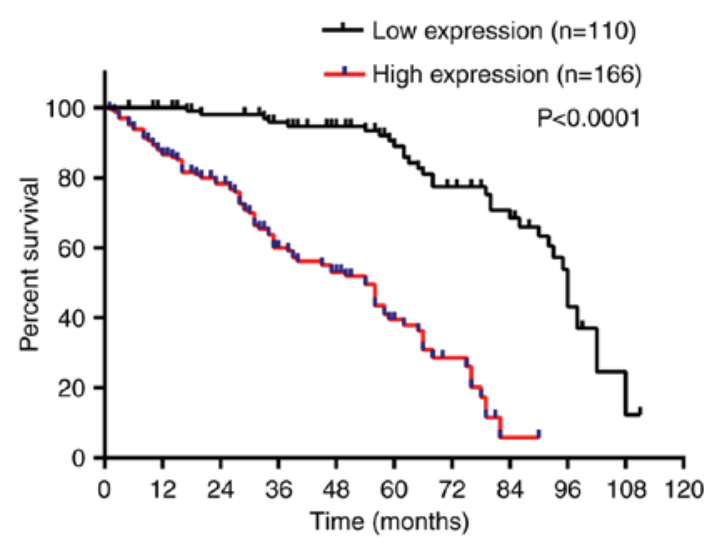

Figure 4. Kaplan-Meier plots of the overall survival of $276 \mathrm{HCC}$ patients, stratified by the expression of UBE2A. Lower expression is indicated by the immunohistochemical score of 0 or 1 , while high expression is indicated by a score of 2 or 3. UBE2A, ubiquitin-conjugating enzyme E2A; HCC, hepatocellular carcinoma.

significant difference was detected between HCC patients with high and low UBE2A expression, as determined according to their immunohistochemical score $(\mathrm{P}<0.0001, \log$-rank test; Fig. 4).

Univariate and multivariate analyses of prognostic parameters in HCC patients. To identify the variables with potential prognostic significance, univariate analysis of each clinicopathological parameter was performed to examine its correlation with the survival of HCC patients. The hazard ratio and P-value for each parameter were used to predict the prognosis of HCC patients. The importance of each parameter was calculated by multivariate Cox proportional hazards model analysis. A stepwise forward-inclusion of clinicopathological parameters in the model was performed through univariate analysis, which revealed that the significant prognostic factors for HCC patients included the UBE2A expression, Histological differentiation, TNM stage and vascular invasion (Table II). Multivariate analysis also yielded the same results as the univariate analysis (Table III).

\section{Discussion}

HCC is a leading cause of cancer-associated mortality worldwide, and tumor recurrence and metastasis are major factors that contribute to the poor prognosis of HCC patients $(29,30)$. Previous studies have confirmed that protein ubiquitination serves multiple roles in different biological processes. It is known that ubiquitination is crucial for protein degradation (31). The E2 ubiquitin-conjugating enzyme RAD6 catalyzes target proteins through ubiquitination (22). The human homologs of yeast DNA repair gene RAD6 are termed UBE2A and UBE2B.

In the present study, it was observed that the expression of UBE2A mRNA and protein was upregulated in HCC, while UBE2A protein was located both in the cytoplasm and nucleus in HCC tissues, although mainly detected in the cytoplasm. qPCR measurement also revealed that UBE2A was upregulated in HCC cell lines compared with the level in normal hepatocytes. Based on these results, it is concluded that UBE2A expression is increased in HCC tissues and cell lines, and may be associated with tumor pathology or prognosis in HCC patients.

UBE2A is a regulator of the G1-S transition and proliferation in human cells by targeting cyclin D1 expression. Cyclin D1 and G1-S transition have been reported to be significantly involved in tumorigenesis (22). In the current study, the UBE2A expression and pathological tumor stage were higher in HCC patients, particularly in patients with vascular invasion, while it was less pronounced in HCC patients with long-term survival. Therefore, it is predicted that UBE2A may affect the development of HCC by regulating the proliferation and migration of HCC cells.

Several studies have been engaged in searching for candidate biomarkers for the treatment and prognosis predition of $\mathrm{HCC}$, identifying AFP, miRNA and long non-coding RNAs as potential biomarkers $(32,33)$. In the present study, among the 276 HCC patients enrolled, UBE2A expression was increased in the cancer tissues compared with the adjacent normal tissues. To illustrate the association between UBE2A expression and the tumor pathology or prognosis, the 276 patients were classified into two groups according to the immunohistochemical score of their tissue specimens. Notably, patients with high UBE2A expression exhibited poor prognosis. All these findings indicated that increased UBE2A expression is associated with the development and progression of HCC, and that UBE2A expression can be used as an independent predictor of prognosis in HCC patients. The overall survival of patients with high UBE2A expression was reported to be reduced in comparison with that of patients with low UBE2A expression according to Kaplan-Meier analysis, which revealed that UBE2A was correlated with the prognosis of HCC. Thus, the prognostic value of UBE2A in 
Table II. Univariate analysis of factors associated with overall survival.

\begin{tabular}{lccr}
\hline Factor & Hazard ratio & 95\% confidence interval & P-value \\
\hline Age (years) & 0.995 & $0.983-1.007$ & 0.383 \\
Sex & 0.970 & $0.740-1.272$ & 0.826 \\
TNM & 1.849 & $1.441-2.371$ & $<0.001$ \\
Histological differentiation & 2.103 & $1.652-2.678$ & $<0.001$ \\
Vascular invasion & 2.147 & $1.642-2.808$ & $<0.001$ \\
Tumor diameter & 0.999 & $0.954-1.046$ & 0.963 \\
UBE2A expression (high vs. low) & 3.415 & $2.572-4.533$ & $<0.001$
\end{tabular}

Cox proportional hazards regression analysis was conducted. TNM, tumor node metastasis; UBE2A, ubiquitin-conjugating enzyme E2A.

Table III. Multivariate analysis of factors associated with overall survival.

\begin{tabular}{lccr}
\hline Factor & Hazard ratio & 95\% confidence interval & P-value \\
\hline Age (years) & 0.994 & $0.982-1.006$ & 0.347 \\
Sex & 0.994 & $0.754-1.311$ & 0.966 \\
TNM & 1.439 & $1.089-1.903$ & 0.011 \\
Histological differentiation & 1.359 & $1.019-1.813$ & 0.037 \\
Vascular invasion & 1.358 & $1.013-1.819$ & 0.041 \\
Tumor diameter & 1.038 & $0.987-1.091$ & 0.146 \\
UBE2A expression (high vs. low) & 2.677 & $1.948-3.680$ & $<0.001$ \\
\hline
\end{tabular}

Cox proportional hazards regression analysis was conducted. TNM, tumor node metastasis; UBE2A, ubiquitin-conjugating enzyme E2A.

HCC was further evaluated, and it was observed that UBE2A was a crucial factor in the prognosis of HCC and would be an independent prognostic biomarker for HCC patients.

However, there were certain limitations to the present study. For instance, there is a lack of data examining the mechanism responsible for the overexpression of UBE2A in HCC tissue, or the substrate that UBE2A degrades in HCC progression. Previous studies have also indicated that UBE2A regulates p53 protein levels by transcriptional and post-transcriptional mechanisms in human cells $(20,34)$. Thus, the mechanism of UBE2A involvement in the development of HCC should be clarified in the future.

In conclusion, the results of the present study suggested that UBE2A serves as an independent prognostic marker for HCC. Furthermore, it was observed that UBE2A expression was associated with the histological differentiation, TNM stage and vascular invasion of patients. All these data indicated that UBE2A serves a critical role in the development of HCC. Thus, elaborating the underlying mechanism of UBE2A in future studies is important, which may offer novel therapeutic targets for HCC.

\section{Acknowledgements}

The authors are grateful to Dr Chun-Yan Gu from the Department of Pathology, Nantong Third People's Hospital, Nantong University (Nantong, China) for her assistance with immunohistochemistry.

\section{Funding}

The present study was supported by grants from the National Natural Science Foundation of China (grant no. 81600449), the Natural Science Foundation of Jiangsu Province (grant no. BK20160420) and Nantong Science and Technology Bureau (grant nos. MS22015105, MS32016025 and YYZ16039).

\section{Availability of data and materials}

The datasets used and analyzed during the current study are available from the corresponding author on reasonable request.

\section{Authors' contributions}

JDS and SZF performed immunohistochemistry and wrote the paper. JDS revised the manuscript. ZLB made substantial contributions to the conception and design of the study. LLJ and YFW performed quantitative polymerase chain reaction and western blotting. FD and ZXL performed statistical analysis. JGS and HZJ performed data collation. All authors read and approved the final manuscript.

\section{Ethics approval and consent to participate}

This study was approved by the Ethics Committee of Nantong Third People's Hospital and all patients provided signed informed consent. 


\section{Consent for publication}

All the patients involved in the study provided signed informed consent for the publication of their data.

\section{Competing interests}

All authors declare that they have no competing interests.

\section{References}

1. Torre LA, Bray F, Siegel RL, Ferlay J, Lortet-Tieulent J and Jemal A: Global cancer statistics, 2012. CA Cancer J Clin 65: 87-108, 2015

2. Jemal A, Bray F, Center MM, Ferlay J, Ward E and Forman D: Global cancer statistics. CA Cancer J Clin 61: 69-90, 2011.

3. Tejeda-Maldonado J, García-Juárez I, Aguirre-Valadez J, González-Aguirre A, Vilatobá-Chapa M, Armengol-Alonso A, Escobar-Penagos F, Torre A, Sánchez-Ávila JF and CarrilloPérez DL: Diagnosis and treatment of hepatocellular carcinoma: An update. World World J Hepatol 7: 362-376, 2015.

4. Chen G, Li X, He G, Yu Z, Luo J, He J and Huang Z: Low expression of GNAI3 predicts poor prognosis in patients with HCC. Int J Clin Exp Med 8: 21482-21486, 2015.

5. Takayama T: Surgical treatment for hepatocellular carcinoma Jpn J Clin Oncol 41: 447-454, 2011.

6. Lau WY, Lai EC and Leung TW: Current role of selective internal irradiation with yttrium-90 microspheres in the management of hepatocellular carcinoma: A systematic review. Int J Radiat Oncol Biol Phys 81: 460-467, 2011.

7. Niu ZS, Niu XJ and Wang WH: Genetic alterations in hepatocellular carcinoma: An update. World J Gastroenterol 22 9069-9095, 2016.

8. van Meer S, de Man RA, Siersema PD and van Erpecum KJ: Surveillance for hepatocellular carcinoma in chronic liver disease: Evidence and controversies. World J Gastroenterol 19: 6744-6756, 2013.

9. Morise Z, Kawabe N, Tomishige H, Nagata H, Kawase J, Arakawa S, Yoshida $\mathrm{R}$ and Isetani M: Recent advances in the surgical treatment of hepatocellular carcinoma. World J Gastroenterol 20: 14381-14392, 2014.

10. Chen X, Liu HP, Li M and Qiao L: Advances in non-surgical management of primary liver cancer. World J Gastroenterol 20 16630-16638, 2014.

11. Zeke A, Misheva M, Remenyi A and Bogoyevitch MA JNK Signaling: Regulation and functions based on complex Protein-protein partnerships. Microbiol Mol Biol Rev 80 793-835, 2016.

12. Cheng CW, Leong KW and Tse E: Understanding the role of PIN1 in hepatocellular carcinoma. World J Gastroenterol 22: 9921-9932, 2016.

13. Zhang A, Yin C, Wang Z, Zhang Y, Zhao Y, Li A, Sun H, Lin D and $\mathrm{Li}$ N: Development and application of a fluorescence protein microarray for detecting serum alpha-fetoprotein in patients with hepatocellular carcinoma. J Int Med Res 44: 1414-1423, 2016.

14. Lok AS, Sterling RK, Everhart JE, Wright EC, Hoefs JC, Di Bisceglie AM, Morgan TR, Kim HY, Lee WM, Bonkovsky HL, et al: Des-gamma-carboxy prothrombin and alpha-fetoprotein as biomarkers for the early detection of hepatocellular carcinoma. Gastroenterology 138: 493-502, 2010.

15. Zhang SY, Lin BD and Li BR: Evaluation of the diagnostic value of alpha-l-fucosidase, alpha-fetoprotein and thymidine kinase 1 with ROC and logistic regression for hepatocellular carcinoma. FEBS Open Bio 5: 240-244, 2015.
16. Hepowit NL, de Vera IM, Cao S, Fu X, Wu Y, Uthandi S, Chavarria NE, Englert M, Su D, Söll D, et al: Mechanistic insight into protein modification and sulfur mobilization activities of noncanonical E1 and associated ubiquitin-like proteins of Archaea. FEBS J 283: 3567-3586, 2016.

17. Kim KI, Chung HK, Park JH, Lee YJ and Kang JH Alpha-fetoprotein-targeted reporter gene expression imaging in hepatocellular carcinoma. World J Gastroenterol 22: 6127-6134, 2016.

18. Zhou Y, Zhao Y, Gao Y, Hu W, Qu Y, Lou N, Zhu Y, Zhang X and Yang H: Hepatitis C virus NS3 protein enhances hepatocellular carcinoma cell invasion by promoting PPM1A ubiquitination and degradation. J Exp Clin Cancer Res 36: 42, 2017.

19. Weissman AM: Themes and variations on ubiquitylation. Nat Rev Mol Cell Biol 2: 169-178, 2001.

20. Chen S, Wang DL, Liu Y, Zhao L and Sun FL: RAD6 regulates the dosage of 553 by a combination of transcriptional and posttranscriptional mechanisms. Mol Cell Biol 32: 576-587, 2012.

21. Lee KY and Myung K: PCNA modifications for regulation of post-replication repair pathways. Mol Cells 26: 5-11, 2008.

22. Cai F, Chen P, Chen L, Biskup E, Liu Y, Chen PC, Chang JF, Jiang W, Jing Y, Chen Y, et al: Human RAD6 promotes G1-S transition and cell proliferation through upregulation of cyclin D1 expression. PLoS One 9: e113727, 2014

23. Bergink $S$ and Jentsch S: Principles of ubiquitin and SUMO modifications in DNA repair. Nature 458: 461-467, 2009.

24. Shekhar MP, Lyakhovich A, Visscher DW, Heng H and Kondrat N: Rad6 overexpression induces multinucleation, centrosome amplification, abnormal mitosis, aneuploidy, and transformation. Cancer Res 62: 2115-2124, 2002.

25. European Association for the study of the liver; European Organisation for Research and Treatment of Cancer: EASL-EORTC clinical practice guidelines: Management of hepatocellular carcinoma. J Hepatol 56: 908-943, 2012.

26. Livak KJ and Schmittgen TD: Analysis of relative gene expression data using real-time quantitative PCR and the 2(-Delta Delta $\mathrm{C}(\mathrm{T})$ ) method. Methods 25: 402-408, 2001

27. Zhou B, Yuan T, Liu M, Liu H, Xie J, Shen Y and Chen P. Overexpression of the structural maintenance of chromosome 4 protein is associated with tumor de-differentiation, advanced stage and vascular invasion of primary liver cancer. Oncol Rep 28: 1263-1268, 2012.

28. Edge SB and Compton CC: The American Joint Committee on cancer: The 7th edition of the AJCC cancer staging manual and the future of TNM. Ann Surg Oncol 17: 1471-1474, 2010.

29. Ni W, Zhang Y, Zhan Z, Ye F, Liang Y, Huang J, Chen K, Chen L and Ding Y: A novel lncRNA uc.134 represses hepatocellular carcinoma progression by inhibiting CUL4A-mediated ubiquitination of LATS1. J Hematol Oncol 10: 91, 2017.

30. Ju LL, Chen L, Li JH, Wang YF, Lu RJ, Bian ZL and Shao JG: Effect of NDC80 in human hepatocellular carcinoma. World J Gastroenterol 23: 3675-3683, 2017.

31. Wang $X$ and Terpstra EJ: Ubiquitin receptors and protein quality control. J Mol Cell Cardiol 55: 73-84, 2013.

32. Yamashita T, Kitao A, Matsui O, Hayashi T, Nio K, Kondo M, Ohno N, Miyati T, Okada H, Yamashita T, et al: Gd-EOB-DTPAenhanced magnetic resonance imaging and alpha-fetoprotein predict prognosis of early-stage hepatocellular carcinoma. Hepatology 60: 1674-1685, 2014.

33. Quagliata L, Matter MS, Piscuoglio S, Arabi L, Ruiz C, Procino A, Kovac M, Moretti F, Makowska Z, Boldanova T, et al: Long noncoding RNA HOTTIP/HOXA13 expression is associated with disease progression and predicts outcome in hepatocellular carcinoma patients. Hepatology 59: 911-923, 2014.

34. Chen S, Wei HM, Lv WW, Wang DL and Sun FL: E2 ligase dRad6 regulates DMP53 turnover in drosophila. J Biol Chem 286: 9020-9030, 2011. 